\title{
INFLUÊNCIA DO TIPO DE DIISOCIANATO E TIPO DE POLIOL NAS PROPRIEDADES DE DISPERSÕES AQUOSAS DE POLIURETANO
}

\author{
V. M. S. de OLIVEIRA ${ }^{1}$, R. LIGABUE ${ }^{2}$ e R. ROCHA ${ }^{2}$ \\ ${ }^{1}$ Universidade Federal Do Rio Grande do Sul, Programa de Pós-Graduação em Engenharia de Minas, \\ Metalúrgica e de Materiais \\ ${ }^{2}$ Pontifícia Universidade Católica do Rio Grande do Sul, Programa de Pós-Graduação em Engenharia \\ e Tecnologia de Materiais \\ E-mail para contato: vitmso@hotmail.com
}

\begin{abstract}
RESUMO - A crescente preocupação com o meio ambiente tem despertado interesse em dispersões aquosas de poliuretano (PUDs), utilizadas em revestimentos, adesivos e selantes, por substituir compostos orgânicos voláteis por água em sua composição. Os parâmetros reacionais alteram as propriedades das PUDs, tais como massa molar, viscosidade e tamanho de partícula. O presente trabalho pretende verificar como a natureza do diisocianato e do poliol influenciam essas propriedades. Para tanto, foram utilizados dois diisocianatos: tetrametilxileno diisocianato (TMXDI) e isoforona diisocianato (IPDI) e diferentes polióis. As PUDs foram sintetizadas pelo processo do prépolímero e caracterizadas. O espectro de infravermelho deste material apresentou banda de carbonila e bandas características do grupo uretano ( $\mathrm{NH}-\mathrm{COO})$. O filme polimérico das PUDs sintetizadas possuem massa molar ponderal média em torno de 20000-100000 $\mathrm{g} / \mathrm{mol}$. A viscosidade obtida das PUDs possui valores entre 5500-100000 mPa.s enquanto o tamanho de partícula médio está entre 72-227 nm.
\end{abstract}

\section{INTRODUÇÃO}

O desenvolvimento de dispersões aquosas de poliuretano (PUDs, do inglês polyurethane dispersions) tem sido motivado pelas novas exigências ambientais que visam reduzir a emissão de compostos orgânicos voláteis (VOCs, do inglês, volatile organic compounds) (Cakić et al., 2010, Garcia-Pacios et al., 2013 e Patel et al., 2010). Esses produtos apresentam muitas vantagens frente aos produtos convencionais base solvente, como por exemplo, a baixa viscosidade e boa aplicabilidade, segundo Cakić et al. (2010), podendo serem empregados em formulações de adesivos, revestimentos (Garcia-Pacios et al., 2013) e tintas base água (Fang et al., 2014).

Os poliuretanos (PUs) são produzidos pela reação de policondensação de um isocianato (di ou polifuncional) com um poliol (polímeros de diversas funções com grupos hidroxila terminais) e outros reagentes como extensores de cadeia e catalisadores. Para que um polímero PU seja disperso em água é necessário inserir, em sua cadeia polimérica, grupos hidrofílicos, que atuam como emulsificante interno. Em água, o efeito estabilizador dos sítios iônicos é devido à formação de diminutas esferas 
que contém um núcleo de segmentos agregados hidrofóbicos em uma camada fronteiriça carregando os grupos iônicos. O resultado é um surpreendente hidrosol ou dispersão aquosa, segundo PérezLimiñana (2005).

Para a síntese de PUDs, uma razão NCO/OH maior que 1,5 é normalmente utilizada, resultando em um pré-polímero com grupos NCO terminais e posteriormente é realizada a extensão de cadeia formando grupos uretano. De acordo com Cakić et al. (2010) extensores de cadeia de baixa massa molar (MM) hidroxilados ou terminados em aminas fornecem importante função na morfologia de fibras poliuretanas, elastômeros, adesivos entre outros. As propriedades de PUDs termoplásticas são geralmente determinadas pela interação entre os segmentos rígidos e flexíveis e pela interação entre os grupos iônicos. Os segmentos flexíveis (i.e. cadeia do poliol) fornecem flexibilidade ao poliuretano, enquanto os segmentos rígidos (formado pela reação entre o isocianato e o extensor de cadeia) são duros e mais polares e possuem menor massa molecular (Garcia-Pacios et al., 2013).

Este trabalho objetivou avaliar a influência dos precursores, diisocianato e poliol, nas propriedades da PUD, tais como, massa molar, viscosidade, tamanho de partícula e distribuição de tamanho de partícula de PUDs.

\section{MATERIAIS E MÉTODOS}

As reações de síntese de dispersões aquosas de poliuretano (PUDs) foram realizadas em um reator de vidro, equipado com agitação mecânica, controle de temperatura, condensador de refluxo e entrada de gás inerte $\left(\mathrm{N}_{2}\right)$. Em todas as reações se utilizou excesso de diisocianato (razão equimolar $\mathrm{NCO} / \mathrm{OH}$ 1,7), $5 \%$ de emulsificante interno, $90 \%$ de extensão de cadeia e $120 \%$ de neutralização. Todos os reagentes foram utilizados sem prévia purificação.

Para avaliar a influência do tipo de diisocianato e tipo de poliol nas propriedades de PUDs, foram sintetizadas cinco PUDs, conforme indica a Tabela 1. Foram utilizados dois diisocianatos, o isoforona diisocianato (IPDI, da Bayer, pureza 99,5\%, funcionalidade 2) e o meta-tetrametilxileno diisocianato (TMXDI, da Cytec, pureza 98,1\%, funcionalidade 2) e quatro tipos de polióis, todos poliésters, cujas características são também mostradas na Tabela 1.

\begin{tabular}{c|c|c|c|c}
\hline Dispersões & Poliol & $\begin{array}{c}\text { MM do poliol } \\
(\mathrm{g} / \mathrm{mol})\end{array}$ & $\begin{array}{c}\text { Funcionalidade } \\
\text { do poliol }\end{array}$ & Diisocianato \\
\hline PUD 1 & Poliéster linear A & 1000 & 2 & TMXDI \\
\hline PUD 2 & Poliéster linear B & 500 & 2 & TMXDI \\
\hline PUD 3 & Poliéster linear C & 2760 & 2,5 & TMXDI \\
\hline PUD 4 & Poliéster ramificado & 2000 & 2 & TMXDI \\
\hline PUD 5 & Poliéster linear A & 1000 & 2 & IPDI \\
\hline
\end{tabular}

Obs.: $\mathrm{MM}=$ massamolar. Proveniências dos polióis poliésteres: A, Degussa; B, King; C, sintetizado e o ramificado, Uniquema. 


\section{9 a 22 de outubro de 2014 \\ Florianópolis/SC}

\subsection{Síntese de PUD}

Em uma reação típica, utilizando o processo do pré-polímero, o reator foi carregado com o poliol poliéster, o emulsificante interno, ácido dimetilol propiônico, DMPA (Rudnik; funcionalidade = 2) e o catalisador dibutil dilaurato de estanho, DBTDL (Miracema-Nuodex Ind.) sob atmosfera inerte.

Atingindo-se a temperatura desejada $\left(80{ }^{\circ} \mathrm{C}\right.$ para TMXDI e $60^{\circ} \mathrm{C}$ para IPDI), foi adicionado lentamente o diisocianato, através de um funil de adição. Após, a temperatura foi elevada até $110{ }^{\circ} \mathrm{C} \mathrm{e}$ procedeu-se a reação fazendo-se o controle do residual de NCO através da titulação com ndibutilamina (Subramani, 2004 e Moss, 1997). A reação foi considerada completa quando o teor de NCO residual foi estabilizado. Em seguida, com a temperatura reduzida para valores abaixo de $40{ }^{\circ} \mathrm{C}$, adicionou-se 5-10\% em massa de acetona para reduzir a viscosidade do meio e, após foi realizada a etapa de neutralização através da adição de trietilamina, TEA. Posteriormente, seguiu-se a etapa da dispersão e extensão de cadeia, que consiste em pesar, em um béquer de $1000 \mathrm{~mL}$, água deionizada e o extensor de cadeia etilenodiamina, EDA (Vetec; pureza $=98 \%$ ), e a essa solução, sob agitação vigorosa $(800-1500 \mathrm{rpm})$, com um funil de adição foi adicionado o pré-polímero, à temperatura próxima da ambiente.

Após a dispersão, os solventes orgânicos foram retirados com o auxílio de um rotovapor ou com uma linha de vácuo até a obtenção do teor de sólidos calculado para a quantidade de água adicionada (teor de sólidos de $40 \%$ ).

\subsection{Técnicas de caracterização}

As PUDs sintetizadas foram caracterizadas por análise de teor de sólidos, ou não voláteis (NV), que consiste em pesar uma pequena amostra antes e depois de colocadas na estufa a $105{ }^{\circ} \mathrm{C}$ por $24 \mathrm{~h}$; medidas de $\mathrm{pH}$, realizadas a $20^{\circ} \mathrm{C}$ em um equipamento QUIMIS modelo $400 \mathrm{~A}$, com eletrodo combinado; viscosidade Brookfield, utilizando um viscosímetro Brookfield DV-II Prime, a $20^{\circ} \mathrm{C}$, utilizando spindles SC4-21, SC4-21 e SC4-29 e com velocidade de rotação de 50 rpm; tamanho de partícula médio e distribuição de tamanho de partícula, realizadas em um Dispersion Analyser LUMiSizer, a $25^{\circ} \mathrm{C}$, utilizando célula quadrática de vidro de $10 \mathrm{~mm}$ x $10 \mathrm{~mm}$, as análises desses resultados foram obtidos com o auxílio do software SEPView. Os filmes das PUDs sintetizadas foram solubilizados em tetraidrofurano (THF) e caracterizados por espectroscopia vibracional na região do infravermelho (IV) em um equipamento Perkin Elmer Instruments Spectrum One FT-IR Spectometer Hair Sampling Acessory, no intervalo de 4000 a $650 \mathrm{~cm}^{-1}$, com o acessório de refletância total atenuada (ATR) utilizando uma célula de seleno de zinco; e cromatografia de permeação em gel (GPC) utilizando um cromatógrafo da Waters Instruments equipado com bomba isocrática 1515 (eluente: THF, fluxo: $1 \mathrm{~mL} / \mathrm{min}$, set de colunas Styragel (temperatura das colunas: $40{ }^{\circ} \mathrm{C}$ ) e detector de índice de refração 2414 (temperatura do detector: $35^{\circ} \mathrm{C}$ ). Esses fimes foram preparados em uma placa de vidro, aplicados com um extensiômetro de $100 \mu \mathrm{m}$ e secos ao ar por uma semana. 


\section{RESULTADOS E DISCUSSÃO}

\subsection{Espectroscopia vibracional no infravermelho}

As PUDs sintetizadas foram caracterizadas através da técnica de espectroscopia vibracional na região do infravermelho (IV), sendo as atribuições das bandas realizadas em comparação aos valores das frequências características para os grupos presentes na molécula, de acordo com a literatura (Silverstein, 1994). A Figura 1 apresenta os espectros de IV típicos de filmes de PUDs sintetizadas com o poliol poliéster A e TMXDI (a) e IPDI (b), respectivamente e na Tabela 2 são apresentadas as atribuições das bandas características para os filmes das respectivas PUDs.
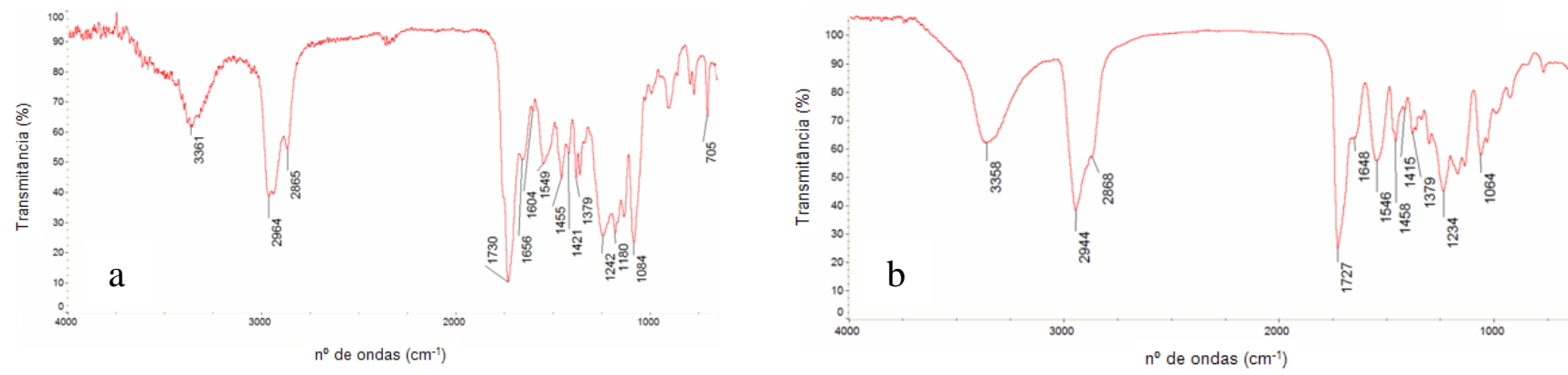

Figura 1. (a) Espectro de IV do filme da PUD 1, sintetizadas com o poliol poliéster linear A e TMXDI e (b) Espectro de IV do filme da PUD 5, sintetizada com o poliol poliéster linear A e IPDI.

Tabela 2. Atribuição das bandas no espectro de IV das PUDs 1 e 5.

\begin{tabular}{|c|c|c|}
\hline PUD 1 & PUD 5 & \multirow{2}{*}{ Atribuição } \\
\hline $\mathrm{n}^{0}$ de ondas $\left(\mathrm{cm}^{-1}\right)$ & $\mathrm{n}^{0}$ de ondas $\left(\mathrm{cm}^{-1}\right)$ & \\
\hline $\begin{array}{c}3361 \\
2964 \text { e } 2865 \\
1730\end{array}$ & $\begin{array}{c}3358 \\
2944 \text { e } 2868 \\
1727\end{array}$ & $\begin{array}{c}v_{\mathrm{s}} \mathrm{NH} \text { (uretano) } \\
v_{\mathrm{as}} \mathrm{CH}_{3} \text { e } v_{\mathrm{s}} \mathrm{CH}_{3} \\
v \mathrm{C}=\mathrm{O} \text { (uretano }+ \text { éster) }\end{array}$ \\
\hline 1656 & 1648 & $\begin{array}{c}v_{\mathrm{as}} \mathrm{COO}^{-}(\mathrm{DMPA})+v \mathrm{C}=\mathrm{O} \\
\text { (uréia) }\end{array}$ \\
\hline 1604 & - & $v \mathrm{C}=\mathrm{C}$ (anel aromático) \\
\hline 1549 & 1546 & $v \mathrm{CN}+\delta \mathrm{NH}$ (uretano) \\
\hline 1455 & 1458 & $\delta_{\mathrm{as}} \mathrm{CH}_{3}$ \\
\hline 1421 & 1415 & $v_{\mathrm{s}} \mathrm{COO}^{-}(\mathrm{DMPA})$ \\
\hline 1379 & 1379 & $\delta_{\mathrm{s}} \mathrm{CH}_{3}$ \\
\hline 1242 & 1234 & $v \mathrm{CO}-\mathrm{O}$ (éster/uretano) \\
\hline 1180 & - & $v \mathrm{C}-\mathrm{O}-\mathrm{C}$ (éster) \\
\hline 1084 & 1084 & $v_{\mathrm{s}} \mathrm{N}-\mathrm{CO}-\mathrm{O}+v \mathrm{C}-\mathrm{O}-\mathrm{C}$ \\
\hline 705 & - & $\omega=\mathrm{C}-\mathrm{H}(\mathrm{TMXDI})$ \\
\hline
\end{tabular}




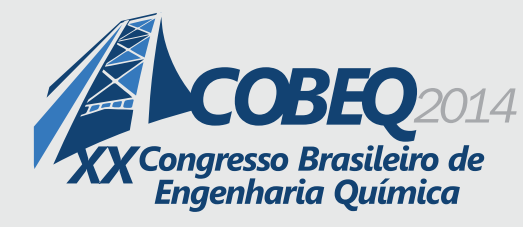

19 a 22 de outubro de 2014
Florianópolis/SC

Os espectros de IV, mostrados nas Figuras 1, referentes aos filmes de PUDs obtidos do poliol poliéster linear A e os diisocianatos TMXDI (a) e IPDI (b), apresentam banda característica da ligação N-H de uretano em $3361 \mathrm{~cm}^{-1}$ (para TMXDI) e $3358 \mathrm{~cm}^{-1}$ (para IPDI). A banda característica do grupo NCO livre residual em torno de $2115 \mathrm{~cm}^{-1}$ não é encontrada nos dois espectros, confirmando a reação total entre os grupos NCO do diisocianato com o poliol e com o extensor de cadeia, o que é desejado, já que NCO livre é tóxico e a existência de NCO livre pode gerar reações secundárias indesejáveis (por exemplo, com a água gerando ácido carbâmico que se decompõe para dar como produto final uréia e biureto, segundo Chattopaddhyay (2007)). Nas regiões de $2964 \mathrm{~cm}^{-1}$ e $2865 \mathrm{~cm}^{-}$ 1, na Figura 1a, e $2944 \mathrm{~cm}^{-1}$ e $2868 \mathrm{~cm}^{-1}$, na Figura 1b, observam-se as bandas características de estiramento assimétrico e simétrico de $\mathrm{CH}_{3}$, respectivamente. Um alargamento na banda atribuída ao estiramento $\mathrm{C}=\mathrm{O}$ (uretano) $\left(1730 \mathrm{~cm}^{-1}\right)$ é observado no espectro apresentado na Figura 1a, em função da sobreposição com a banda de estiramento $\mathrm{C}=\mathrm{O}$ de grupo éster. No espectro apresentado na Figura $1 \mathrm{~b}$ essa banda deslocou-se para $1727 \mathrm{~cm}^{-1}$. Outra banda característica pode ser observada em 1656 $\mathrm{cm}^{-1}$ (Figura 1a) e $1648 \mathrm{~cm}^{-1}$ (Figura 1b) referente ao estiramento assimétrico $\mathrm{COO}^{-}$proveniente do DMPA e estiramento $\mathrm{C}=\mathrm{O}$ devido à uréia. Em $1421 \mathrm{~cm}^{-1}$ (Figura 1a) e $1415 \mathrm{~cm}^{-1}$ (Figura 1b) aparece uma banda de baixa intensidade característica do estiramento simétrico $\mathrm{COO}^{-}$do DMPA. As bandas características das ligações $(\mathrm{CO}-\mathrm{O})$ e $(\mathrm{C}-\mathrm{O}-\mathrm{C})$ de poliéster ocorrem em $1242 \mathrm{~cm}^{-1}$ e $1180 \mathrm{~cm}^{-1}$, respectivamente, na Figura 1a. Na Figura 1b, a banda de estiramento em $1234 \mathrm{~cm}^{-1}$ pode ser atribuída à ligação (CO-O) de uretano. Na região de $1084 \mathrm{~cm}^{-1}$ (Figura 1a) e $1064 \mathrm{~cm}^{-1}$ (Figura 1b) tem-se uma banda de média intensidade característica do grupo N-CO-O de uretano (Otts, 2005). Em 1604 e $705 \mathrm{~cm}^{-1}$ (Figura 1a) aparecem duas bandas de baixa intensidade características, respectivamente, ao estiramento da ligação $\mathrm{C}=\mathrm{C}$ e a deformação fora do plano da ligação $\mathrm{C}=\mathrm{C}-\mathrm{H}$ de anel aromático. Essas duas últimas bandas não são evidentes na Figura 1b, já que na PUD sintetizadas com IPDI não existe anel aromático.

\subsection{Teor de sólidos, pH, massa molar, tamanho de partícula e viscosidade}

A Tabela 3 apresenta os resultados das análises de teor de sólidos, $\mathrm{pH}$, cromatografia de permeação em gel (GPC), que fornece a massa molar numérica média (Mn), a massa molar ponderal média (Mw) e o índice de polidispersão (IP), tamanho de partícula (T.P.) e viscosidade das PUDs sintetizadas.

Tabela 3. Resultados das análises.

\begin{tabular}{cccccccc}
\hline Dispersão & $\mathrm{NV}(\%)$ & $\mathrm{pH}$ & $\begin{array}{c}\mathrm{Mn} \\
(\mathrm{g} / \mathrm{mol})\end{array}$ & $\begin{array}{c}\mathrm{Mw} \\
(\mathrm{g} / \mathrm{mol})\end{array}$ & I.P. & $\begin{array}{c}\text { T.P. } \\
(\mathrm{nm})\end{array}$ & $\begin{array}{c}\text { Viscosidade } \\
(\mathrm{mPa} . \mathrm{s})\end{array}$ \\
\hline PUD 1 & 45 & 8 & 23065 & 44228 & 1,92 & 227 & 19500 \\
\hline PUD 2 & 42 & 8 & 12490 & 22706 & 1,82 & 99 & 18800 \\
\hline PUD 3 & 38 & 9 & 32243 & 73480 & 2,28 & 212 & 84000 \\
\hline PUD 4 & 44 & $*$ & 17714 & 35638 & 2,01 & 163 & $>100000$ \\
\hline PUD 5 & 35 & 9 & 36670 & 103756 & 2,83 & 72 & 5500 \\
\hline
\end{tabular}

NV: não voláteis, I.P.: índice de polidispersão, T.P.: tamanho de partícula. *Análise não realizada devido à alta viscosidade. 


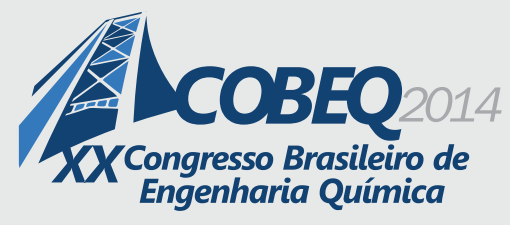

19 a 22 de outubro de 2014

Florianópolis/SC

As dispersões sintetizadas apresentaram teor de sólidos (não voláteis) entre 35 e $45 \%$. O teor de sólidos influencia muito a viscosidade da dispersão. De acordo com a literatura, o aumento da viscosidade nas dispersões PU pode ser explicado, em parte, pelo alto teor de sólidos, onde para valores $\geq 42 \%$ a viscosidade aumenta fortemente. Este fato pode ser explicado pelo comportamento típico de formação de estrutura fractal ou gel, que é atribuída à forte interação entre as partículas, levando a um empacotamento das mesmas (Nanda, 2006 e Madbouly, 2005). Além do teor de sólidos, parâmetros como tamanho de partícula médio e massa molar do poliol utilizado na síntese de PUDs também apresentam influência em sua viscosidade. Os pHs das dispersões ficaram em torno de 8 e 9 , dentro da faixa descrita na literatura para formar uma dispersão estável (Koutsky, 1970). Esses pHs são levemente alcalinos devido a adição de excesso de TEA na etapa da neutralização dos grupos iônicos.

Tipo de poliol: Dentre as PUDs sintetizadas com TMXDI, o polímero PU de maior massa molar (MM) foi obtido com o poliol poliéster linear C, de $\mathrm{MM}=2760 \mathrm{~g} / \mathrm{mol}$ (PUD 3) mostrando, como era de se esperar, que quanto maior a MM do poliol utilizado maior será a MM do polímero PU formado.

Os valores de viscosidade destas PUDs aumentam com o aumento da massa molar do poliol, como consequência de um aumento na MM do PU formado (Tabela 3). Para a PUD 3, onde se utilizou o poliol poliéster linear $\mathrm{C}(\mathrm{MM}=2760 \mathrm{~g} / \mathrm{mol})$ a alta viscosidade encontrada deve ser devido à formação de partículas grosseiras, pois, geralmente, há uma ampla e heterogênea distribuição de tamanho de partícula médio (alta polidispersidade) em termos de volume na dispersão. Isto pode explicar a viscosidade excessiva do pré-polímero durante a etapa de dispersão, onde a dispersão do pré-polímero se torna mais difícil produzindo partículas alongadas (Pérez-Liminãna, 2005). Este fato é confirmado pelo alto valor de tamanho de partícula médio encontrado para esta PUD. Outro fator que auxilia no aumento da MM do polímero PU na PUD 3 é a funcionalidade maior do poliéster linear C (2,5), possibilitando a formação de uma rede polimérica de mais alta massa molar, o que é confirmado pelo valor do Mw desta PUD (73480 g/mol), bastante superior ao valor de Mw das demais PUDs, sintetizadas com TMXDI e diferentes poliós.

A PUD 4, sintetizada com o poliol poliéster ramificado $(\mathrm{MM}=2000 \mathrm{~g} / \mathrm{mol})$, apresentou um Mw menor (35638 g/mol) que as PUDs sintetizadas com poliésteres lineares, como a PUD 1 (44228 $\mathrm{g} / \mathrm{mol}$ ), sintetizada com poliol de $\mathrm{MM}=1000 \mathrm{~g} / \mathrm{mol}$. Entretanto, o tamanho de partícula diminuiu e a viscosidade aumentou bruscamente na PUD 4. Este fato pode ser explicado através de dois efeitos relacionados com a estrutura do póliol: (a) quanto menor o tamanho de partícula, maior a viscosidade de PUDs; (b) as ramificações do poliéster podem influenciar negativamente no crescimento da cadeia e na movimentação dessas cadeias, dificultando o fluxo, ou seja, aumentando a viscosidade, conforme dados descritos na literatura (Subramani, 2004 e Nanda, 2006). O índice de polidispersão (I.P.) é bastante próximo em todas as PUDs, o que significa que o fato de diferentes MM ou se estes são lineares ou ramificados não altera significantemente a relação Mw e Mn.

Tipo de diisocianato: A PUD 5, sintetizada com o diisocianato IPDI, apresentou valor de massa molar ponderal média significativamente maior $(103756 \mathrm{~g} / \mathrm{mol})$ que todas as outras dispersões $(22706$ - $73480 \mathrm{~g} / \mathrm{mol}$ ), sintetizadas com TMXDI, confirmando a maior reatividade do IPDI. Grupos receptores de elétrons ligados ao grupamento $\mathrm{NCO}$ aumentam a reatividade dos diisocianatos e os 


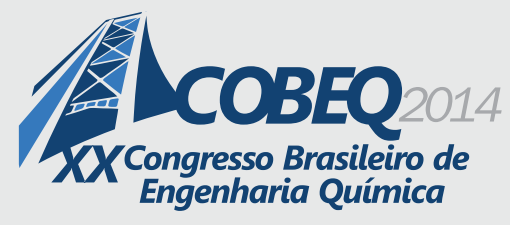

19 a 22 de outubro de 2014
Florianópolis/SC

doadores a reduzem, por isso diisocianatos aromáticos são mais reativos do que os alifáticos (Oliveira, 2008). O TMXDI não é considerado diisocianato aromático, pois os grupos NCO não estão diretamente ligados ao anel. Substituintes volumosos próximos do sítio de reação diminuem a velocidade da reação. Assim, os grupos NCO do IPDI que são ligados a átomos de carbono primário e secundário possuem reatividades diferentes de acordo com Vilar (2005). O TMXDI é um diisocianato terciário e, devido ao impedimento estérico dos grupos NCO ligados ao átomo de carbono, possui baixa reatividade, notavelmente com a água, o que possibilita seu emprego em sistemas reativos dispersos em água (Vilar, 2005). A PUD 5, sintetizada com IPDI, apresentou o menor tamanho de partícula $(72 \mathrm{~nm})$, sendo indicada para aplicações onde se requer profunda penetração no substrato. Tamanhos de partícula maiores são preferíveis para facilitar a secagem rápida, como é o caso da PUD $1(227 \mathrm{~nm})$.

Os resultados de tamanho de partícula médio das PUDs sintetizadas com TMXDI e diferentes polióis estão dados, também na Tabela 3, porém os valores apresentado são valores médios. Uma informação também importante é a distribuição do tamanho de partícula. É notável a ampla e heterogênea distribuição de tamanho de partícula médio em todas as PUDs sintetizadas, o que influencia significativamente na viscosidade da dispersão produzida. Este fato é confirmado na literatura, que apresenta uma maior heterogeneidade na distribuição de partículas em termos de volume, e essa maior dispersão pode ser devida a excessiva viscosidade do pré-polímero durante a etapa de dispersão na produção da partícula iônica, produzindo partículas com formas mais alongadas, as quais são responsáveis pelas diferentes medidas de tamanho de partícula (Pérez-Limiñana, 2005).

\section{CONCLUSÃO}

A PUD 5, sintetizada com o diisocianato IPDI apresentou maior massa molar ponderal média (103756 g/mol), devido a maior reatividade do IPDI quando comparado com o diisocianato TMXDI. Além disso, a PUD 5 apresentou o menor valor de tamanho de partícula e, contrário ao esperado, uma viscosidade relativamente baixa, o que pode ser atribuído ao menor teor de sólidos dessa PUD (35\%). As PUDs sintetizadas com TMXDI possuem massa molar ponderal média em torno de 35000-73000 $\mathrm{g} / \mathrm{mol}$. Os valores de massa molar foram, em geral, maiores para polímeros PU produzidos a partir de polióis de maior massa molar, levando a uma maior cadeia de segmentos flexíveis no polímero disperso e menores quando utilizado poliol de menor massa molar ou ramificado.

\section{REFERÊNCIAS}

CAKIĆ, S. M.; RISTIĆ, I. S.; DJORDJEVIĆ, D. M.; STAMENKOVIĆ, J. V.; STOJILJKOVIĆ, D. T. Effect of the chain extender and selective catalyst on thermooxidative stability of aqueous polyurethane dispersions. Progress in Organic Coating, v. 67, p. 274-280, 2010.

CHATTOPADHYAY, D. K.; RAJU, K. V. S. N. Structural engineering of polyurethane coatings for high performance applications. Progress in Polymer Science, v. 32, p. 352-418, 2007.

FANG, C.; ZHOU, X.; YU, Q.; LIU, S.; GUO, D.; YU, R.; HU, J. Synthesis and characterization of low crystalline waterborne polyurethane for potential application in water-based ink binder. Progress in Organic Coatings, v. 77, p. 61-71, 2014. 
GARCÍA-PACIOS, V.; JOFRE-RECHE, J. A.; COSTA, V.; COLERA, M.; MARTÍN-MARTÍNEZ, J. M. Coatings prepared from waterborne polyurethane dispersions obtained with polycarbonates of 1,6-hexanediol of different molecular weights. Progress in Organic Coatings, v. 76, p.1484-1493, 2013.

KOUTSKY, J. A.; HIEN, N. V.; COOPER, S. L. Some results on electron microscope investigations of polyether-urethane and polyester-urethane block copolymers. Polymer Letters, v. 8, p 353359, 1970.

MADBOULY, S. A. et al. Rheological behavior of aqueous polyurethane dispersions: Effects of solid contend, degree of neutralization, chain extension, and temperature. Macromolecules, v. 38, p. 4014-4023, 2005.

NANDA, A.; WICKS, D. The influence of the ionic concentration, concentration of the polymer, degree of neutralization and chain extension on aqueous polyurethane dispersions prepared by the acetone process. Polymer, v. 47, p. 1805-1811, 2006.

OLIVEIRA, V. M. S. Síntese e caracterização de dispersões aquosas de poliuretano. Porto Alegre, 2008. 109 p. Dissertação de Mestrado em Engenharia e Tecnologia de Materiais. Faculdade de Engenharia, Pontifícia Universidade Católica do Rio Grande do Sul, Brasil.

OTTS, D.; URBAN, M. Heterogeneous crosslinking of waterbourne two-component polyuretanes (WB 2K-PUR); stratification processes and the role of water. Polymer, v. 46, p. 2699-2709, 2005.

PATEL, A.; PATEL, C.; PATEL, M.G.; PATEL, M.; DIGHE, A. Fatty acid modified poluyretane dispersion for surface coatings: Effect os fatty acid contend and ionic contend. Progress in Organic Coatings, v. 67, p. 255-263, 2010.

PÉREZ-LIMIÑANA, M. A. et al. Characterization of waterborne polyurethane adhesives containing different amounts of ionic groups. International Journal os Adhesion \& Adhesives, v. 25, p. 507-517, 2005.

SILVERSTEIN, R. M.; BASSLER, G. C.; MORRIL, T. C. Identificação Espectrométrica de Compostos Orgânicos. Rio de Janeiro: Guadanabara, 1994.

SUBRAMANI, S.; CHEONG, I. W.; KIM, J. H. Chain extension of water-borne polyurethanes from methyl ethyl ketoxime/E-caprolactam-blocked aromatic isocyanates. Progress in organic coatings, v. 51, p. 329-338, 2004.

VILAR, W. Química e tecnologia dos poliuretanos. 3a ed. Rio de Janeiro: Vilar Consultoria, 2005. $400 \mathrm{p}$. 